\title{
Ethnobotanical Use and Conservation of Plants Biodiversity by the Local Community of Welkait Wereda, Western Tigray, Ethiopia
}

\author{
Fitsumbirhan Tewelde $^{1 *} \quad$ Mebrahtom Mesfin ${ }^{2}$ \\ 1.Ethiopian Biodiversity Institute, Forest and Rangeland Plants Biodiversity, Mekelle Center, Ethiopia \\ 2.Ethiopian Biodiversity Institute, Crop and Horticultural Biodiversity, Mekelle Center, Ethiopia
}

\begin{abstract}
An ethonobotanical study is important from the standpoint of conservation and sustainable use of community based knowledge plant biodiversity. A survey to identify and document local community knowledge and a conservation status of the plant biodiversity was conducted in welkait wereda, western Tigray, Ethiopia. A reconnaissance survey, plant interview and different ranking methods were applied to gather primary ethnobotanical data collection. Respondents from the age of 15-80 years old were selected with the help of Wereda and Tabia agricultural experts and local elders. Data was analyzed using ranks, percentages and SPSS version 20. Nearly 97 plant species serve for different ethnobotanical use that belongs to 52 families and 86 genera were identified from welkait wereda. The growth patern of the plant species were 39\% tree, $31 \%$ herb, $28 \%$ shrub and $2 \%$ climbers. $41 \%$ of the remedy prepared from the leaves, $16 \%$ from root, $19 \%$ stem, $15 \%$ fruit, and the remaining is another part of the plant species. The habitat of the identified medicinal plant was $90 \%$ wild and $10 \%$ domesticated. Agricultural expansion, firewood collection and free grazing were the three most threats to medicinal plants as described by the informants. Anogeissus leiocarpa, Terminalia brownie and Securidaca longipedunculata were the most threatened medicinal plants based on the score given by the key informants. Education and age of the informants positively correlate $(p<0.001, p<0.005$ respecively) with number of medicinal plants mentioned. Community based awareness creation and insitu and exsitu conservation method need to be implemented for sustainable utilization of plant biodiversity.
\end{abstract}

Keywords: Adi remets, Welkait, medicinal, Conservation, threats

DOI: $10.7176 /$ ALST/83-01

Publication date: November $30^{\text {th }} 2020$

\section{INTRODUCTION}

Forests are indispensable for human existence and well-being as well as for survival of two thirds of all terrestrial animals and plant species (Lal and Junior, 2011). They provide us with food, oxygen, shelter, recreation, spiritual sustenance and they are the source for over 5,000 commercially traded products, ranging from pharmaceuticals to timber and clothing (SCBD, 2010). Accordingly, ethnobotanical researches and applications are mostly done around natural ecosystems (Sebukeera, 2010) such as forests, grasslands, woodlands, and wetlands as well as in many other micro-habitats, from where the prosperity of ethnobotanical knowledge is retrieved. Forest are used in the manufacture of a great many medicinal plant products and pharmaceuticals as well as nutritional supplements, while these products were obtained through a systematic ethnobotanical research. In addition, (Hanazaki et al., 2006) point out the main aspect investigated from forest ethnobotany is the use and investigations of numerous medicinal plants as well as development of ethnopharmacological studies. (Ibrar et al., 2007, Colfer et al., 2006) added that a forest is a constant source of maintaining genetic diversity of plants and animals and these resources are available to humans, from which ethnobotanical researches would be applicable. Farmers and forest dwelling people possess a great deal of indigenous knowledge arising from their utilization of forest resources. Forests are the source of many hidden treasures and a fertile ground for scientific research (Sebukeera, 2010). Furthermore; forests serve as both a classroom and a source of raw materials for local health practitioners (Balick and Cox, 1996). As (Thomas et al, 1989) Specified that forest and the benefits they afford in the form of wood, food, income, and watershed protection has an important and critical role in facilitating people to secure a stable and adequate food supply. Accordingly, ethnobotanical studies and applications were done on accumulated people's experiences on these different use categories of different plant biodiversity resources.

The Assessment of woody species for timber, medicine and other uses in the forests showed that the species richness of medicinal species was highest (Hanazaki et al., 2006, Bisht and Badoni, 2009). These medicinal richness circumstances are very important for the concentration of ethnobotanical research and applications about their values. Furthermore, forests have been increasingly seen as serving multiple functions (Hamilton et al, 2003). for example, forests and woodlands of Ethiopia act as sources of raw materials for wood-based energy ( $70 \%$ from the total energy needed), construction, wood-based industries, farm implements, fuel-wood and employment opportunities and other non-timber forest products such as coffee, spices, honey medicinal products, 
animal fodder, wild-edible plants (fruits, seeds, leaves, roots, tubers) etc. From these manifold use values of forest biodiversity resources, different types of ethnobotanical useful values of plants in different parts of the country, Ethiopia, have been taking place although it is not in all places. In the absence of forests, ethnobotanical research as well as application might not be available (Demel Teketay, 2001). Systematic study and application of indigenous knowledge is important for sustainable use of resources and sustainable development (Thomas, 1995). Biodiversity and traditional knowledge of its various properties and uses have long provided and continue to provide vital resources for medicine discovery and health care (SCBD, 2010). Recent information indicates that limited ethnobotanical studies have been conducted in the catchment area of the Mekelle biodiversity center, particularly Tigray region to document the use of medicinal plants. However, no such study has so far been conducted in welkait wereda. Therefore, the protection, documentation of forest and rangeland plants biodiversity and associated community knowledge are compulsory, and it is on the basis of this gap that the present study was undertaken. More ever, the main objective of this survey was to study an ethnobotanical use and management practice of medicinal plants and the associated indigenous knowledge in the study wereda. Specifically, this study aims to;

$>\quad$ Identify types of plant species used for different use by community of the study wereda

$>$ Pinpoint the habits and habitat distribution plant species in the study wereda.

$>$ Recognize threats to medicinal plants and management practice in the study wereda

$>$ Determine the dependency of the local community in plant biodiversity resource

\section{Material and method \\ Description of the Study Area}

Welkait wereda administration is found in the western zone of Tigray regional state northwestern Ethiopia. It is bordered to the north by Kafta Himora and to the south by Tsegede; it is also bordered on the east by the weredas of Tahtay Adiyabo and Asgede Tsimbla, to the northeast, on the other side Tekeze River, and Tselemti is to the east. Adi Remets is the administrative center of the wereda figure 1. Based on the census conducted by the Central Statistical Agency of Ethiopia (CSA, 2007), Welkait wereda has a total population of 138,926, of whom 70,504 are men and 68,422 women; 10,758 or $7.74 \%$ are urban residents. With an area of $3,374.52 \mathrm{~km}^{2}$, Welkait has a population density of 41.17 people $/ \mathrm{km}^{2}$. A total of 30,375 households were counted in this wereda, resulting in an average of 4.57 persons in a household, and 29,336 housing units. The majority of the inhabitants $(97.28 \%)$ practiced Ethiopian Orthodox Christianity, with reporting that as their religion, while $2.71 \%$ of the populations were Muslim (CSA, 2007).

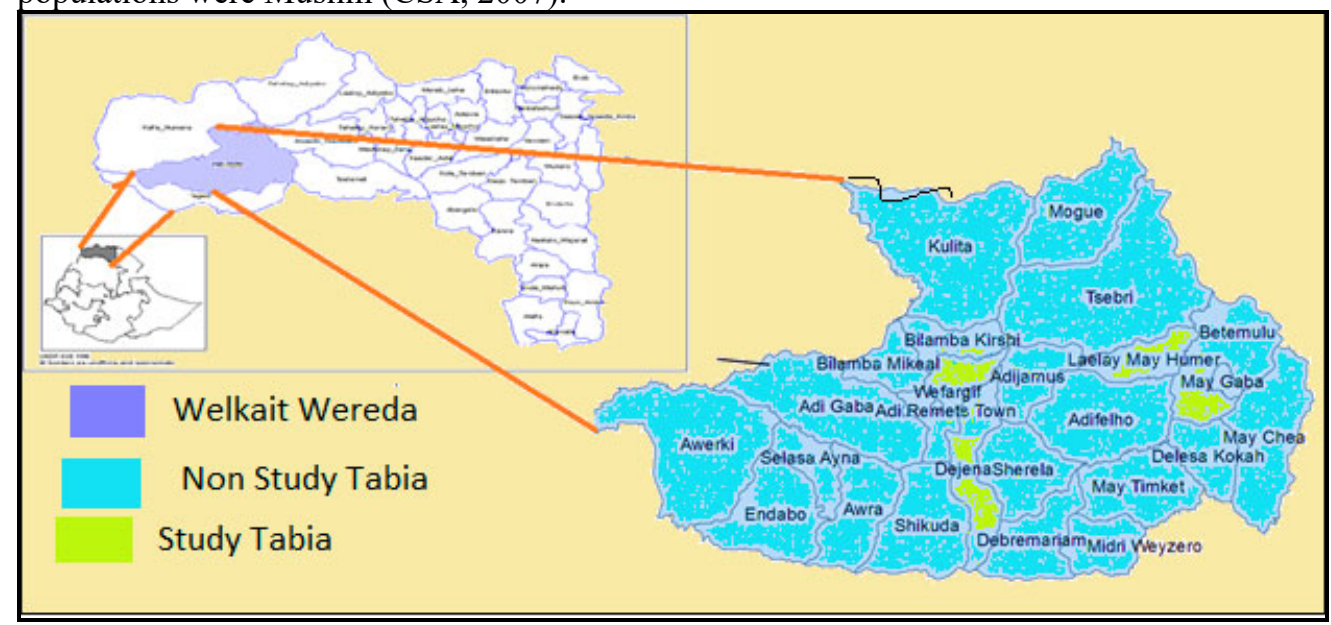

Figure 1: Map of Welkait Wereda

\section{Design and Site Selection}

Rapid ethnobotanical appraisal approach (REA) design was employed to conduct an ethnobothanical study of plants in welkayt wereda. By using this research design, small sample size and area was taken to sketch out how the community acts as a whole. A reconnaissance survey of the study area was conducted in May, 2019. A total of 5 Tabias was selected for ethnobotanical data collection based on availability of traditional healers identified with the assistance of local authorities, elders and knowledgeable persons.

\section{Selection of Informants}

Selection of informants was done following (Martin, 1995) which indicated that when recording indigenous knowledge held by knowledgably traditional healers or by certain social groups the choice of key informant is critical. The age of informants was grouped into less than 30, 30-40 and greater than 40 intervals. From each site 
of Kebele, 3-4 individual key informants was purposely selected from each study sites by using information and recommendations from the Wereda agricultural office officials, local kebele administrators and kebele agricultural officials, knowledgeable elders and religious leaders as well as the local community.

\section{Ethnobotanical Data Collection}

Ethnobotanical data were collected by the method semi-structured interview following (Martin, 1995) and (Cotton, 1996), field observation and group discussion. Most of the interviews and discussions were conducted in the local language of the Wereda (Tigrigna), which is a common language in the study area and the Tigrai region as well. The necessary information about the plants such as habit, habitat, were recorded.

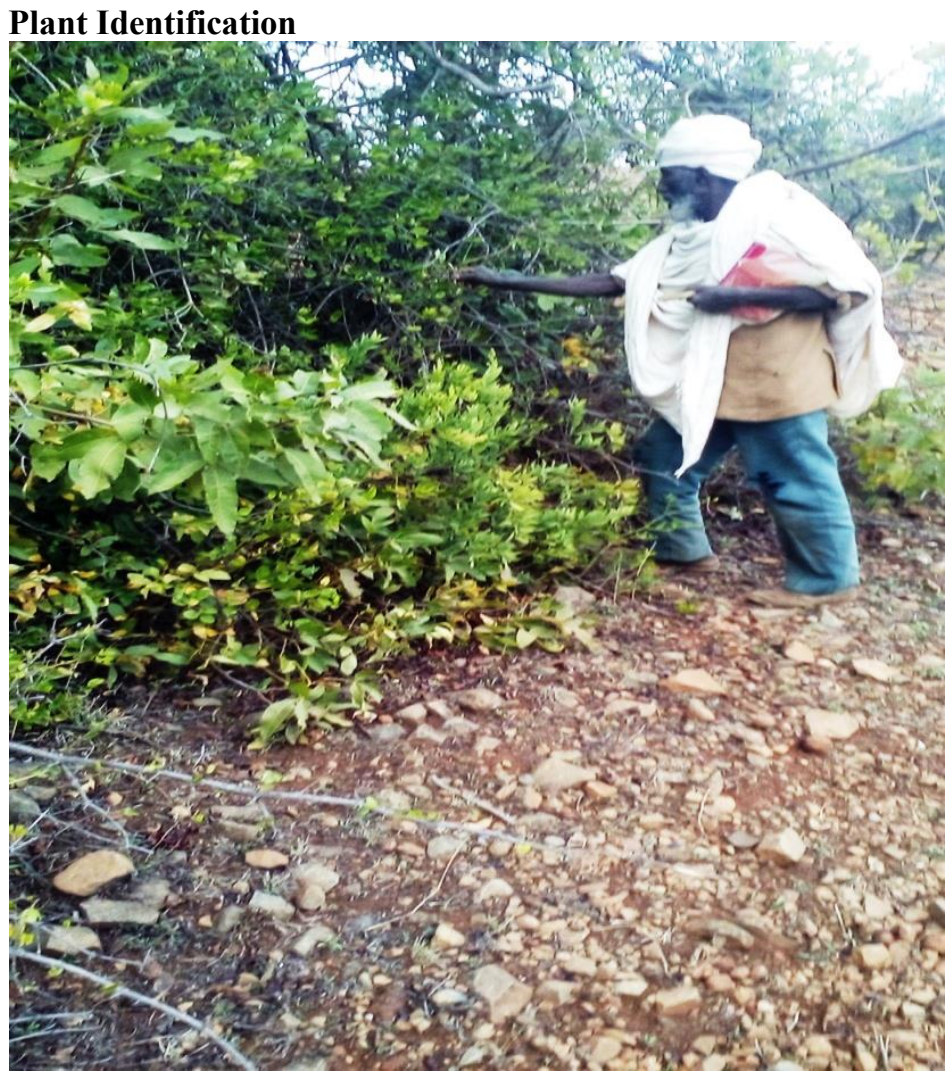

The identification works of some common and well known species were made in the field. Further identification was performed by using the Natural Data base for Africa (NDA, 2008) software, illustrations and taxonomic keys and published volumes of the Flora of Ethiopia and Eritrea (Hedberg and Edwards, 1989, Edwars et al., 1995, Edwars et al, 2000, Hedberg et al., 2003,). Besides, plant interview method was used in order to confirm the emic classification (classification of the local community) of the informants with the ethic classification (scientific classification) system as shown in Picture 1

Picture 1; plant interview method at Dejena field with key informant (photo by Fitsumbirhan T.)

The key informants were asked to show the local name given for each plant species as well as for what type of use can it serve. Besides, when we got plant species which is not mentioned by the respondents during discussion, we asked them to tell the ethnobotanical use of those plants.

\section{Informant Consensus}

In order to evaluate the reliability of information recorded during the interview, informants were communicated at least two times for the same ideas and the legitimacy of the information was proved and recorded. Later, if the thought of the informant digresses from the original information, it was excluded since it was reflected inapt gen. Only the relevant ones were taken into account and statistically analyzed.

\section{Preference Ranking}

Following (Martin, 1995), preference ranking was made for five medicinal plants for the most frequently encountered disease in the study area and for which they have several alternative plants as readily. Ten preferentially selected key informants were participated in this exercise. The informants were gave the plants and ask to arrange the five medicinal plants based on their personal preference of effectiveness. The medicinal plant that was believed to be the most effective were gave the highest value i.e. 5, and the one with the least effectiveness received the lowest value i.e. 1. Based on the total score of each species the rank were determined, and this helped to indicate the most effective medicinal plants used by the community to treat commonly occurred illness.

\section{Direct Matrix Ranking}

Direct matrix ranking were conducted following (Cotton, 1996) for 7 multiuse medicinal plants commonly report by informants. Based on the relative benefits obtained from each plant, 10 informants were favorably selected and asked to give value to each characteristic. The list of attributes included medicinal, fire wood, building (construction), charcoal, furniture, edible fruits and fence. The scores were add in order to compare use values of 
medicinal plants and help to identify the main causes of over harvesting of the medicinal plants so that to pinpoint recommended conservation measure for sustainability of that species.

\section{Ranking of Threats to Plants Resource}

Ranking of threats to medicinal plants report by most of the informants in the study area were accompanied using 10 selected key informants as defined by (Martin, 1995, Alexiades, 1996). Furthermore, five main threats were select and the informants were ask to give seven for the most threatening factor and one for the least threatening factor based on their personal thought in the study area. This information was used to determine the highest threats to traditional medicinal plants in the area and helps to propose the necessary appropriate conservation measures, and for sustainably use of forest and range land plants biodiversity.

\section{Ranking of Threatened Plants Resource}

Using the method applied by (Martin, 1995), ranking of five medicinal plants that report by the informants as threatened in the study area were conduct with ten key informants (knowledgeable traditional healers). The preferentially selected informants were give the names of five traditional medicinal plant species considered threatened in the study area and arrange the plants based on the degree of threat /scarcity by assigning 5 for the most threatened and 1 for the least threatened plant species. Finally, the scores of each species were summed and ranked. This information was help to identify the most threatened species so that appropriate conservation measures would suggest.

\section{Data Analysis}

Ethnobotanical information collected using different ethnobotanical methods such as questionnaire survey and interviews, along with the data in the form of scores were organize, enter and analyze in Microsoft Excel. Data was subject to expressive analysis and percentages were generated. The data from ranking methods (direct matrix ranking, preference/priority ranking) were present in the form of ranks. Ranks were determined based on the total scores under each trait.

\section{Result and discussion}

\section{Demographic back ground of informants}

The demographic condition of the informants was assessed using the terminology age group, educational level and gender. Accordingly most of the informants $(69 \%)$ were male informants and the remaining $(31 \%)$ were from female informants. Besides, the age of respondents were grouped in to three categories. The highest percent of the informants were from the middle age group (from30-40). The other demographic condition assessed during the study in the area was the educational status of the informants. Moreover, Most of the informants, $64 \%$ were those that didn't able to read and write. Very few respondents scored secondary educational level (9-12) and the remaining was scored primary (1-8) level of education as shown in the following figure 2 .

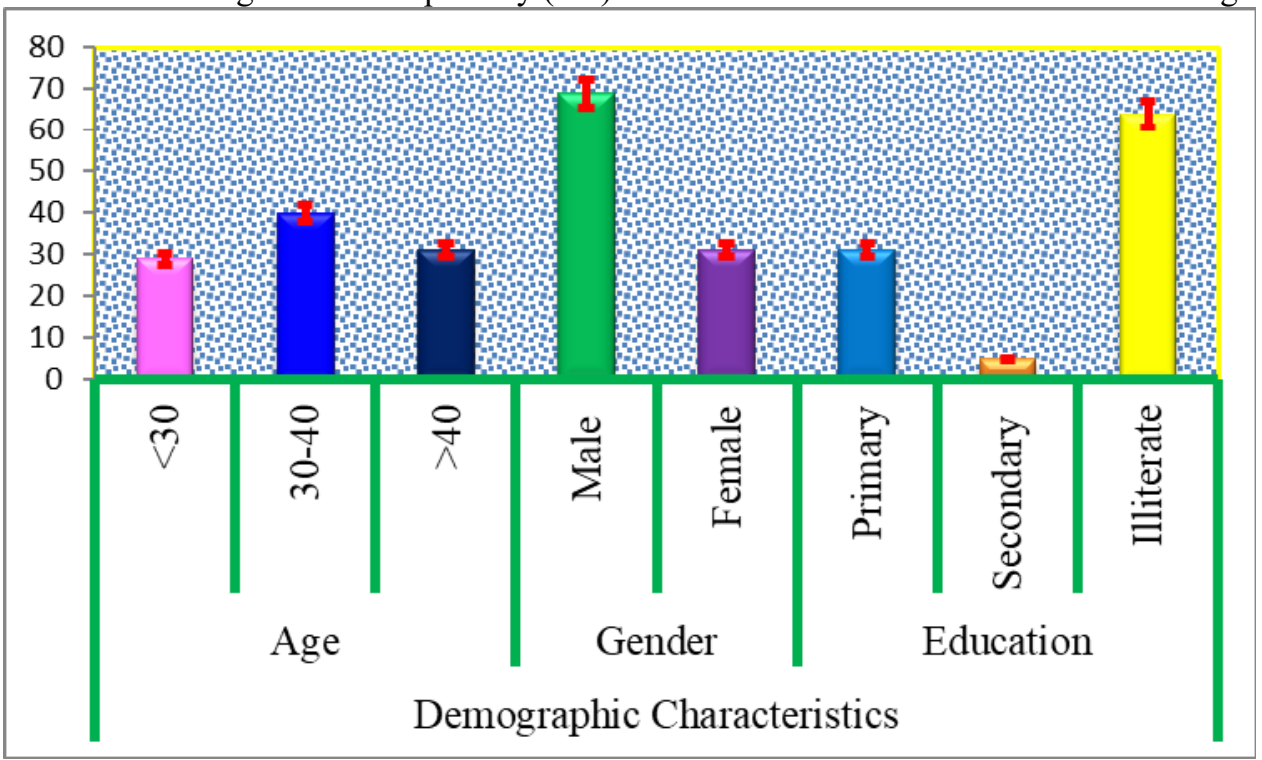

Figure 2; Demographic Status of informants

Floristic diversity of medicinal plant

A total of 97 medicinal plant biodiversity resources belonging to 52 families and 86 genera were documented 
from Welkait Wereda, Western Tigray region of Ethiopia. Fabaceae is the leading family from the ethnobothanicaly useful plants mentioned by the local community in the study area. This is a good indicator of the fact that, the people of the study district highly dependent in plant biodiversity resource utilization since the ancient time for different use values. In line with this finding, other researchers reported that Fabaceae is the leading family with the highest number of medicinal plants in various diseases treatment in Ethiopia and somewhere in the world (Buzuneh et al., 2018, Alfred, 2011, Rahman, 2013, Andarge et al., 2015, Lulekal et al., 2008, Regassa, 2013). Similarly, (Lulekal et al., 2008) noted that Asteraceae is the dominant one among others. The growth form of the plants includes 39\% tree, $31 \%$ herb $28 \%$ shrub and 2\% climbers. Similar results were reported by (Chekole, 2017, Gidey et al., 2015, Kebede, 2016, Mesfin et al., 2009, Hailemariam et al., 2009, Eskedar Abebe .2011) as trees were the most dominant growth form. The part of the plant that used for different use value includes $41 \%$ leave, $16 \%$ root, $15 \%$ stem, $15 \%$ fruit, and the remaining belong to other parts as revealed in table 1 below. Other studies conducted elsewhere in Ethiopia also showed (Mersha, 2011, Tamene, 2011, Ashagre, 2011, Asnake et al., 2016, Chekole et al., 2015, Maryo et al., 2015) leaves was the major plant part used for remedy preparations.

\section{Ecological status of the plants}

The habitat distribution of the medicinal plants in Welkait wereda show more than $90 \%$ are wild habitat and the remaining $10 \%$ only is cultivated. This entails that the majority of plants of medical importance are not yet cultivated properly by traditional healers or other concerned bodies. Thus, the high dependency on medicinal plants which are collected from the wild habitats may have a significant impact on the forthcoming accessibility of these resources and it likely account for their susceptibility to being overexploited (Andarge et al, 2015). Based on the informants' perception, most plant species was used to treat for human disease than livestock. This study also agrees with the finding of (Assegid and Tesfaye, 2014, and Abiyu et al., 2014) in which most species were used to treat human ailments than livestock ailments. This result indicates that the local communities mostly used medicinal plants to treat human ailments than livestock ailments. It is an indication of high prevalence of human disease than livestock in the area.The ecological status of the mentioned medicinal plants in study area was, $52 \%$ found rarely, $40 \%$ moderately and $8 \%$ in an abundant condition. In terms of the condition used a remedy for medication fresh, dry and both dry and fresh were the dominant method of preparation respectively as indicated in the following figure below. The use of fresh material is more curative than dry as suggested by (Abera, 2014, Mesfin et al., 2014, Kefalew et al., 2015, Lamorde et al., 2010).
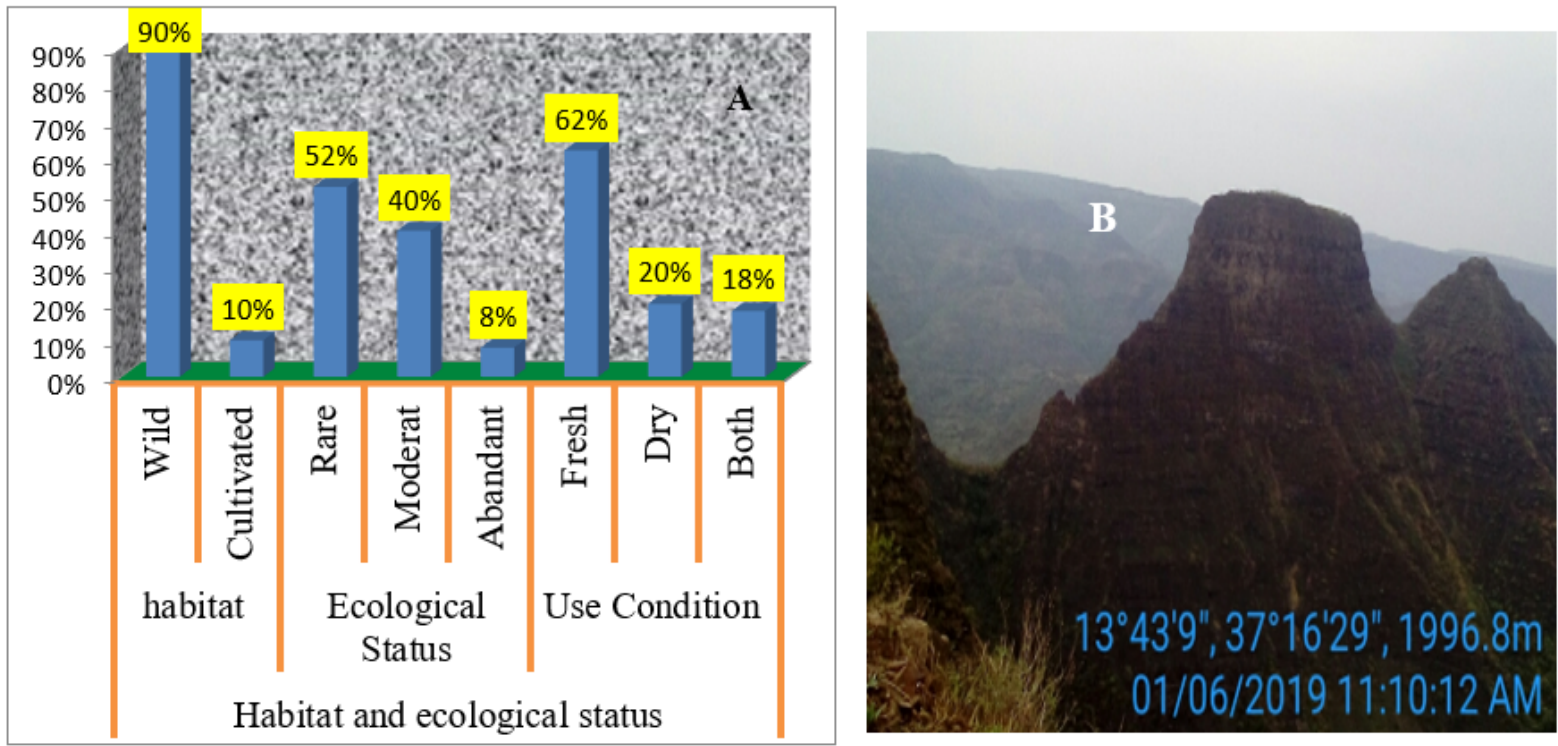

Figure 3; A=Habitat and Ecological Status of Medicinal Plants, B=web photo of Welkait wereda

\section{Route of administration and application}

For every remedy it has its own way of application and route of administration. Based on this inkling, the respondents in the study district asked to mention which route of administration and application were used for each type of remedy preparations. Accordingly, oral ranked as the highest route of remedy administration followed by dermal by route of administration respectively as shown in the following figure 4 below. This result was similar to the findings of previous investigators (Teklehaymanot and Giday, 2007) reported as oral application was the dominant route of administration.

The prepared traditional medicines were applied in a number of methods, among which drinking (45\%), 
eating $(25 \%)$, smearing $(5 \%)$, smoking $(7 \%)$, and others $(18 \%)$ were mentioned the dominant meted of application. In this study, drinking and eating application account for the largest percentage.

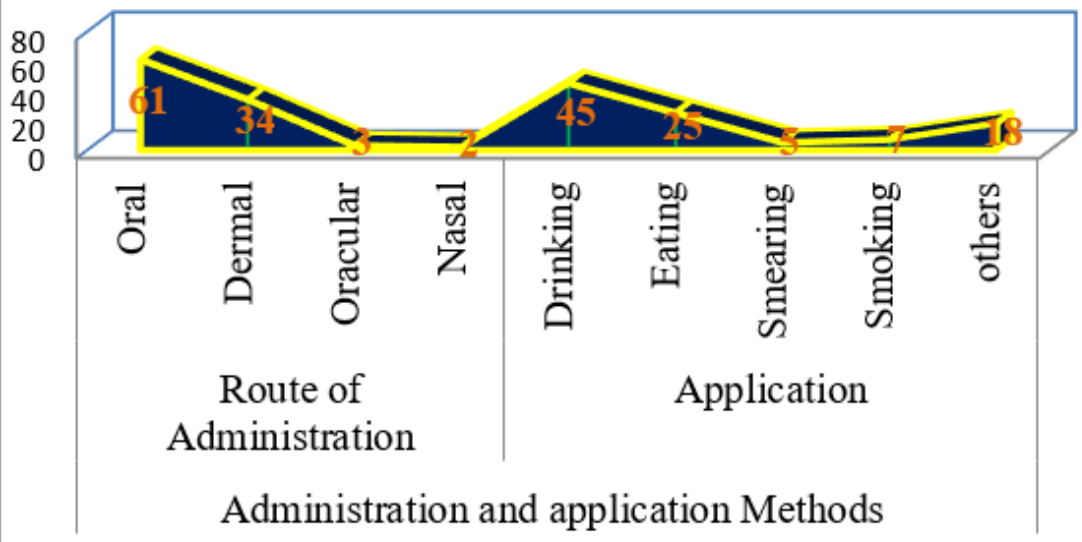

Figure 3; Route of application and administration

Table 1; Floristic Diversity and use value of Plants from Welkait Wereda

\begin{tabular}{|c|c|c|c|c|c|c|c|}
\hline Plant scientific name & Family name & local name & Habit & Use part & Disease treated/use & $\begin{array}{l}\text { Condi } \\
\text { tion }\end{array}$ & Method \\
\hline $\begin{array}{l}\text { Diospyros mespiliformis } \\
\text { Hochst. ex A. DC. }\end{array}$ & Ebenaceae & Aye & $\mathrm{T}$ & $\begin{array}{l}\text { Fruit } \\
\text { Stem } \\
\text { Stem } \\
\end{array}$ & $\begin{array}{l}\text { Food } \\
\text { Bloating* } \\
\text { Ornamental }\end{array}$ & $\begin{array}{l}\text { F } \\
\text { D } \\
D\end{array}$ & Pounding, drinking \\
\hline $\begin{array}{l}\text { Grewia ferruginea Hochst. ex A. } \\
\text { Rich. }\end{array}$ & Malvaceae & Tsinquayt & $S$ & Leave & Fire burn & $F$ & Smearing \\
\hline Agave sisalana & Agavaceae & Eka & $\mathrm{H}$ & Latex & Ear defect & $\mathrm{F}$ & Squeezing \\
\hline Allium sativum L. & Alliaceae & $\begin{array}{l}\text { Tsaeda } \\
\text { shigurti/etse pprie }\end{array}$ & $\mathrm{H}$ & Bulb & Impotence & $\mathrm{F}$ & Crushing \& smearing \\
\hline $\begin{array}{l}\text { Lannea fruticosa (Hochst. ex } \\
\text { A. Rich.) Eng }\end{array}$ & Anacardiaceae & Dugdugunga & $\mathrm{T}$ & bark & Evil eye & $\mathrm{B}$ & Smoking \\
\hline Rhus natalensis & Anacardiaceae & Swarya/Tetaele & $S$ & $\begin{array}{l}\text { leaves } \\
\text { stem }\end{array}$ & $\begin{array}{l}\text { Fishing } \\
\text { Tooth brush }\end{array}$ & $\begin{array}{l}F \\
F\end{array}$ & Decoction \\
\hline Schinus molle & Anacardiaceae & Tikur berbere & $\mathrm{T}$ & Leaves & insect repellant & $\mathrm{F}$ & Decoction \\
\hline Anethum graveolens L. & Apiaceae & Shilan & $\mathrm{H}$ & Whole & Asthma & $\mathrm{B}$ & Crushing \& drinking \\
\hline $\begin{array}{l}\text { Acokanthera schimperi } \\
\text { (A. DC.) Schweinf. }\end{array}$ & Apocynaceae & Mebtie & S & Fruit latex & Wart & $\mathrm{F}$ & Cramming \\
\hline $\begin{array}{l}\text { Calotropis procera (Aiton) W.T. } \\
\text { Aiton }\end{array}$ & Apocynaceae & Gindae & $S$ & stem/root & Haemoredin & $F$ & $\begin{array}{l}\text { Crushing \& and } \\
\text { cramming }\end{array}$ \\
\hline Carissa spinarum L. & Apocynaceae & Agam & $S$ & Latex & Wound healing & $\mathrm{F}$ & Cramming the latex \\
\hline Tagetes minuta L. & Asteracea & Etsefarus & $\mathrm{H}$ & Whole & Evil eye & $\mathrm{B}$ & Fumigate \\
\hline Artemisia afra Jacq. ex willd. & Asteraceae & Tsihtet & $\mathrm{H}$ & leaf & Evil eye & $\mathrm{B}$ & Smelling \\
\hline Echinops Kebericho Mesfin & Asteraceae & Dander & $S$ & Whole & Evil eye & $\mathrm{D}$ & Fumigating \\
\hline Lactuca sativa L. & Asteraceae & Selata & $\mathrm{H}$ & Leave & Night blindness & $\mathrm{F}$ & Crushing \& eating \\
\hline Pluchea dioscoridis (L.) DC. & Asteraceae & Shitene & $S$ & Leave & Anti-vomit & $\mathrm{F}$ & Pounding \& smelling \\
\hline $\begin{array}{l}\text { Stereospermum kunthianum } \\
\text { Cham. }\end{array}$ & Bignoniaceae & Argzana & $\mathrm{T}$ & Bark & wound healing & $\mathrm{F}$ & $\begin{array}{l}\text { Crushing and } \\
\text { decoction the paste }\end{array}$ \\
\hline Adansonia digitata L. & Bombacaceae & Dima & $\mathrm{T}$ & Fruit & Stomach ache & $\mathrm{D}$ & Grounding \\
\hline Cordia africana Lam. & Boroginaceae & Akui & $\mathrm{T}$ & $\begin{array}{l}\text { Stem } \\
\text { Fruit } \\
\text { Fruit } \\
\end{array}$ & $\begin{array}{l}\text { Construction } \\
\text { Food } \\
\text { Amoeba }\end{array}$ & $\begin{array}{l}\mathrm{D} \\
\mathrm{F} \\
\mathrm{F} \\
\end{array}$ & $\begin{array}{l}\text { Grinding \& } \\
\text { swallowing }\end{array}$ \\
\hline Brassica rapa L. & Brassicaceae & Hamli adri & $\mathrm{H}$ & Leaves & wound & $\mathrm{F}$ & Crushing \& Tie \\
\hline
\end{tabular}




\begin{tabular}{|c|c|c|c|c|c|c|c|}
\hline Plant scientific name & Family name & local name & Habit & Use part & Disease treated/use & $\begin{array}{l}\text { Condi } \\
\text { tion }\end{array}$ & Method \\
\hline Lepidium sativum L. & Brassicaceae & Shinfae & $\mathrm{H}$ & Seed & Dysentery** & $\mathrm{B}$ & Orally drinking \\
\hline $\begin{array}{l}\text { Boswellia papyrifera (Del.) } \\
\text { Hochst. }\end{array}$ & Burseraceae & Meker & $\mathrm{T}$ & Latex & Wound & $\mathrm{D}$ & Smearing \\
\hline Boscia angustifolia A. Rich & Capparidaceae & Kermed & $S$ & Leaves & Livestock ticks & $\mathrm{F}$ & Decoction \\
\hline $\begin{array}{l}\text { Boscia senegalensis } \\
\text { (Pers.) Lam. ex Poir. }\end{array}$ & Capparidaceae & Hamta & $\mathrm{S}$ & Bark & water purification & $\mathrm{D}$ & Grounded \\
\hline Capparis tomentosa Lam. & Capparidaceae & Andel & S & Root & Fibril illness & $\mathrm{F}$ & $\begin{array}{l}\text { Fumigating \& inhaling } \\
\text { of the smoke }\end{array}$ \\
\hline Maerua angolensis DC. & Capparidaceae & Keremo & $\mathrm{T}$ & Stem & Milk flavor \& curdle & $\mathrm{B}$ & Smoking \\
\hline Carica papaya L. & Caricaceae & Papaya & $\mathrm{H}$ & Leaves & Wound* & $\mathrm{F}$ & Crushing and smearing \\
\hline $\begin{array}{l}\text { Maytenus arbutifolia (A. Rich.) } \\
\text { Wilczek }\end{array}$ & Celastraceae & Atat & S & Root & itching/scabies & $\mathrm{F}$ & Boil and wash. \\
\hline $\begin{array}{l}\text { Maytenus senegalensis (Lam.) } \\
\text { Exell }\end{array}$ & Celastraceae & Argudi & $\mathrm{T}$ & Bark & Stomach pain & $\mathrm{F}$ & Grinding \& drink \\
\hline Cissus petiolata & Chenopodiaceae & Alke & $\mathrm{H}$ & Stem & Fibril illness & B & Tie in the neck \\
\hline $\begin{array}{l}\text { Anogeissus leiocarpa (DC.) } \\
\text { Guill. \& Perr. }\end{array}$ & Combretaceae & Hanse & $\mathrm{T}$ & $\begin{array}{l}\text { Bark } \\
\text { Branches } \\
\text { Stem } \\
\text { Stem } \\
\text { Leave }\end{array}$ & $\begin{array}{l}\text {-Abdominal } \\
\text { Pain } \\
\text {-Fence } \\
\text {-Firewood } \\
\text {-Construction } \\
\text {-Forage }\end{array}$ & $\begin{array}{l}\text { F } \\
\text { B } \\
\text { D } \\
\text { D } \\
\text { F }\end{array}$ & -Crushing \& drinking \\
\hline $\begin{array}{l}\text { Combretum molle } \\
\text { R.Br. ex G.Don }\end{array}$ & Combretaceae & Akuma & $\mathrm{T}$ & Stem & Fumigation & $\mathrm{D}$ & Smoking \\
\hline Terminalia brownii Fresen. & Combretaceae & Weyba & $\mathrm{T}$ & $\begin{array}{l}- \text { Stem } \\
\text {-Stem }\end{array}$ & $\begin{array}{l}\text {-Fibril illness } \\
\text {-Butterfly }\end{array}$ & $\mathrm{D}$ & $\begin{array}{l}\text { Vaporization } \\
\text { Fumigating }\end{array}$ \\
\hline Vernonia amygdalina Delile & Asteraceae & Gzawa & $S$ & Root & abdominal pain & $\mathrm{D}$ & Pounding \& drinking \\
\hline Cucumis dipsaceus Ehrenb. & Cucurbitaceae & Hafaflo & $\mathrm{H}$ & Root & Abdominal pain & $\mathrm{F}$ & Decoction \\
\hline Cucumis pustulatus Hook. f. & Cucurbitaceae & Lemin bayta & $\mathrm{H}$ & Root & Abdominal cramp & $\mathrm{F}$ & Grinding \&eating \\
\hline Cucurbita pepo L. & Cucurbitaceae & Hamham & $\mathrm{H}$ & Fruit & Evil eye & $\mathrm{D}$ & Fumigating \\
\hline Sansevieria erythraeae Mattei & Dracenaceae & Trm eka & $\mathrm{H}$ & Leaf & Ear defect & $\mathrm{F}$ & $\begin{array}{l}\text { Squeezing \& taking } \\
\text { oracularlly based }\end{array}$ \\
\hline Euclea divinorum Hiern. & Ebenaceae & etse patos/gibara & $S$ & Fruit & evil sprite & $\mathrm{F}$ & Fumigating \\
\hline Tragia uncinata M. Gilbert & Euphorbiacea & Amae & $\mathrm{H}$ & Root & Blood clotting & $\mathrm{F}$ & $\begin{array}{l}\text { Squeezing \& } \\
\text { cramming }\end{array}$ \\
\hline Croton macrostachyus Del. & Euphorbiaceae & Tambok & $\mathrm{T}$ & Leaves & Scabies & $\mathrm{B}$ & Crushing \\
\hline Euphorbia tirucalli L. & Euphorbiaceae & Qinchib & $\mathrm{T}$ & Latex & Warts & $\bar{F}$ & Cramming with butter \\
\hline Flueggia virosa & Euphorbiaceae & Harmazo & $S$ & Leaf & Bloating* & $\mathrm{F}$ & decoction \\
\hline Parkinsonia aculeate L. & Fabacea & Shewit hagay & $\mathrm{T}$ & Leaves & Bloating in goat & $\mathrm{F}$ & eating \\
\hline Acacia polyacantha Willd. & Fabaceae & Gumero & $\mathrm{T}$ & $\begin{array}{l}\text { Bark } \\
\text { Stem } \\
\text { Branches } \\
\text { Stem } \\
\text { Leaves } \\
\end{array}$ & $\begin{array}{l}\text {-bladder pain } \\
\text {-Construction } \\
\text {-Fence } \\
\text {-Timber } \\
\text {-Forage }\end{array}$ & B & $\begin{array}{l}\text { Crushing, squeezing } \\
\text { and drinking the liquid }\end{array}$ \\
\hline Acacia seyal Del. & Fabaceae & Keyh chea & $\mathrm{T}$ & Gum & Fibril illness & $\mathrm{D}$ & $\begin{array}{l}\text { Tie in the neck as a } \\
\text { necklace }\end{array}$ \\
\hline Calpurnia aurea (Aiton) Benth. & Fabaceae & Hitsawtsi & S & Leave & Parasite & $\mathrm{F}$ & $\begin{array}{l}\text { Crushing, squeezing \& } \\
\text { spraying }\end{array}$ \\
\hline $\begin{array}{l}\text { Desmodium barbatum } \\
\text { (L.) Benth. }\end{array}$ & Fabaceae & Balenga qolu' & $\mathrm{T}$ & Leaf & Arthritis & $\mathrm{F}$ & Crushing \& covering \\
\hline $\begin{array}{l}\text { Dichrostachys cinerea } \\
\text { Wight et Arn. }\end{array}$ & Fabaceae & Goneq & $\mathrm{T}$ & Stem & Sprain & $\mathrm{F}$ & Tie \\
\hline $\begin{array}{l}\text { Faidherbia albida } \\
\text { (Delile) A. Chev. }\end{array}$ & Fabaceae & Momona & $\mathrm{T}$ & Leave & Anti dandruff & $\mathrm{F}$ & Crushing and smearing \\
\hline Ficus ovate Vahl & Moraceae & Beles adgi & $S$ & Latex & Wound* & $\mathrm{F}$ & Cramming \\
\hline
\end{tabular}




\begin{tabular}{|c|c|c|c|c|c|c|c|}
\hline Plant scientific name & Family name & local name & Habit & Use part & Disease treated/ use & $\begin{array}{l}\text { Condi } \\
\text { tion }\end{array}$ & Method \\
\hline Trigonella foenum-graecum L & Fabaceae & Abaake & $\mathrm{H}$ & Seed & Abdominal pain & $\mathrm{F}$ & grounded \\
\hline $\begin{array}{l}\text { Becium grandiflorum } \\
\text { var. obovatum }\end{array}$ & Lamiaceae & Tebeb & $\mathrm{H}$ & Leaves & Wound healing & F & crushed \\
\hline $\begin{array}{l}\text { Clerodendrum myricoides } \\
\text { (Hochst.) R. Br. Ex Vatke }\end{array}$ & Lamiaceae & Surbetri & $S$ & Leaves & Epilepsy & $\mathrm{F}$ & $\begin{array}{l}\text { Crush, squeeze and } \\
\text { drink }\end{array}$ \\
\hline Salvia nilotica Jacq. & Lamiaceae & Cheguri awald & $\mathrm{H}$ & Root & Snake bite & $\mathrm{F}$ & Crushing \& drinking \\
\hline Linum usitatissimum L. & Linaceae & Entatie & $\mathrm{H}$ & Seed & Constipation & $\mathrm{D}$ & $\begin{array}{l}\text { Decoction } \\
\text { \&powdering drinking }\end{array}$ \\
\hline Buddleia polystachya & Loganiaceae & Metere & $\mathrm{T}$ & Leaves & Fire burn & $\mathrm{F}$ & Cramming the paste \\
\hline Sida ovata Forssk & Malvaceae & Deqi daer0 & $\mathrm{H}$ & Root & Wound & $\mathrm{F}$ & Crushing \& Cramming \\
\hline Azadirachta indica A. Juss. & Meliaceae & $\mathrm{Nim}$ & $\mathrm{T}$ & Leave & poultry parasite & $\mathrm{F}$ & $\begin{array}{l}\text { Crushing and } \\
\text { squeezing and drink } \\
\text { the liquid }\end{array}$ \\
\hline Albizia amara (Roxb.) Boiv. & Fabaceae & Chigono & $\mathrm{T}$ & Bark & Arthritis & $\mathrm{F}$ & Decoction \\
\hline Ficus sycomorus L. & Moraceae & Sagla & $\mathrm{T}$ & $\begin{array}{l}\text { Fruit } \\
\text { Leaves } \\
\text { Stem } \\
\text { Bark }\end{array}$ & $\begin{array}{l}\text { Edible } \\
\text { fire burn } \\
\text { Firewood } \\
\text { Fiber }\end{array}$ & $\begin{array}{l}\text { F } \\
F \\
D \\
F\end{array}$ & Crushing \& cramming \\
\hline Ficus thonningii Blume & Moraceae & shibaka & $\mathrm{T}$ & $\begin{array}{l}\text { Bark } \\
\text { leave }\end{array}$ & $\begin{array}{l}\text { Abdominal } \\
\text { Fodder }\end{array}$ & $\begin{array}{l}F \\
F\end{array}$ & Crushing \\
\hline Ficus vasta Forssk. & Moraceae & Daero & $\mathrm{T}$ & $\begin{array}{l}\text { Fruit } \\
\text { Latex }\end{array}$ & $\begin{array}{l}\text { Edible } \\
\text { wart }\end{array}$ & $\begin{array}{l}\mathrm{D} \\
\mathrm{F}\end{array}$ & Smearing \\
\hline Eucalyptus globules Labill & Myrtaceae & $\begin{array}{l}\text { Tsaeda } \\
\text { kalamit } \\
\text { OS } \\
\end{array}$ & $\mathrm{T}$ & Leaves & Evil spirit, cough & $\mathrm{F}$ & $\begin{array}{l}\text { Boil it in water and } \\
\text { inhale its vapor. }\end{array}$ \\
\hline $\begin{array}{l}\text { Syzygium guineense (Willd.) } \\
\text { DC }\end{array}$ & Myrtaceae & Liham & $\mathrm{T}$ & Fruit & $\begin{array}{l}\text { Intestinal } \\
\text { blooding } \\
\end{array}$ & F & $\begin{array}{l}\text { Grinding and } \\
\text { swallowing }\end{array}$ \\
\hline Ximenia americana L. & Olacaceae & Mlo & $\mathrm{T}$ & $\begin{array}{l}\text {-Fruit } \\
\text {-Fruit }\end{array}$ & $\begin{array}{l}\text { Intestinal parasite } \\
\text {-Food }\end{array}$ & $\begin{array}{l}-F \\
-F\end{array}$ & $\begin{array}{l}\text { Crushing, squeezing } \\
\text { and drinking }\end{array}$ \\
\hline $\begin{array}{l}\text { Jasminum abyssinicum } \\
\text { Hochst. ex DC. }\end{array}$ & Oleaceae & shaferro & $\mathrm{T}$ & Leaves & Abdominal pain & $\mathrm{F}$ & Grinding and drinking \\
\hline Argemone mexicana L. & Pappavaraceae & Eshok/dander & $\mathrm{H}$ & Latex & Wound * & $\mathrm{F}$ & Cramming \\
\hline Sesbania quadrata & Pedaliaceae & Saspanya & $\mathrm{H}$ & leaves & Cattle disease* & $\mathrm{F}$ & Pounding and eating \\
\hline Phytolacca dodecandra L'Hér. & Phytolaccaceae & Shibtie & $\mathrm{C}$ & Leave & Rabies & $\mathrm{F}$ & Drinking \\
\hline Plumbago zeylanica L & Plumbaginaceae & Aftuh & $S$ & -Root & $\begin{array}{l}\text {-Swelling } \\
\text {-Abdominal pain }\end{array}$ & $\begin{array}{l}\mathrm{F} \\
\mathrm{F}\end{array}$ & Crushing and smearing \\
\hline Premna oligotricha Baker & Lamiaceae & Sasa & $\mathrm{C}$ & Leaves & Bloating & $\mathrm{F}$ & $\begin{array}{l}\text { Pounding, and } \\
\text { smearing }\end{array}$ \\
\hline $\begin{array}{l}\text { Securidaca longipedunculata } \\
\text { Fresen. }\end{array}$ & Polygalaceae & Shitera & $S$ & $\begin{array}{l}\text { Stem } \\
\text { Bark } \\
\text { Bark } \\
\text { Root } \\
\text { Stem } \\
\text { Root } \\
\end{array}$ & $\begin{array}{l}\text {-Fibril illness } \\
\text {-Abdominal pain } \\
\text {-evil eye } \\
\text {-evil sprit } \\
\text { - snake bite } \\
\text {-Mosquito replant }\end{array}$ & $\begin{array}{l}\text { F } \\
\text { F } \\
\text { F } \\
\text { B } \\
\text { F } \\
D\end{array}$ & $\begin{array}{l}\text {-Fumigating } \\
\text {-Grinding } \\
\text { Decoction } \\
\text { Pounding } \\
\text { Crushing \&smearing } \\
\text { Fumigating } \\
\end{array}$ \\
\hline Rumex abyssinicus Jacq. & Polygonaceae & Moqmoqo & $\mathrm{H}$ & Root & Blood pressure & $\mathrm{D}$ & $\begin{array}{l}\text { Grounded \& taking } \\
\text { with spices orally }\end{array}$ \\
\hline $\begin{array}{l}\text { Rumex nervosus } \\
\text { Vahl. } \\
\end{array}$ & Polygonaceae & hehot & S & Leafy stem & Food \& amoebic & $\mathrm{F}$ & Grinding \&eating \\
\hline Rhamnus prinoides L'Herit. & Rhamnaceae & Ghesho & $S$ & Leaves & Tonsil & $\mathrm{F}$ & Grinding \\
\hline Ziziphus abyssinica & Rhamnaceae & Gaba-agdi & $\mathrm{T}$ & Leaves & Anti dandruff & $\mathrm{F}$ & Crushing \& decoction \\
\hline Ziziphus spina-christi (L.) Desf. & Rhamnaceae & Gaba & $\mathrm{T}$ & $\begin{array}{l}\text {-Fruit } \\
\text {-leaves }\end{array}$ & $\begin{array}{l}\text {-Food } \\
\text {-Dandruff }\end{array}$ & $\mathrm{D}$ & Pounding \& cramming \\
\hline Rosa abyssinica & Rosaceae & roz & $S$ & Fruit & against hookworm & $\mathrm{F}$ & Decoction \\
\hline Spermacoce sphaerostigma & Rubiaceae & Tinigita & $\mathrm{H}$ & Whole & Evil sprit & $\mathrm{F}$ & Pounding \& smearing \\
\hline
\end{tabular}




\begin{tabular}{|c|c|c|c|c|c|c|c|}
\hline Plant scientific name & Family name & local name & Habit & Use part & Disease treated/use & $\begin{array}{l}\text { Condi } \\
\text { tion }\end{array}$ & Method \\
\hline Citrus limon (L.) Burm.f. & Rutaceae & Lemin & $\mathrm{T}$ & Fruit & evil eye/evil sprit & $\mathrm{F}$ & fumigating \\
\hline Ruta chalepensis L. & Rutaceae & Chena adam & $\mathrm{H}$ & Leaves & Milk flavor & $\mathrm{F}$ & Crushing \\
\hline Dodonaea angustifolia L. f. & Sapindaceae & Tahses & $S$ & Leaves & Sore * & B & $\begin{array}{l}\text { Crushing and } \\
\text { cramming }\end{array}$ \\
\hline $\begin{array}{l}\text { Mimusops kummel } \\
\text { Bruce ex A.DC }\end{array}$ & Sapotaceae & Kummel & $\mathrm{T}$ & $\begin{array}{l}\text {-Fruit } \\
\text {-Fruit }\end{array}$ & $\begin{array}{l}\text {-Abdominal pain } \\
\text {-Food }\end{array}$ & $\begin{array}{l}-F \\
-F\end{array}$ & $\begin{array}{l}\text { Crushing, mix with } \\
\text { better and eat with } \\
\text { Injera }\end{array}$ \\
\hline Pennisetum villosum & Scrophulariaceae & Chira kurkur & $\mathrm{H}$ & Root & Eye disease & $\mathrm{F}$ & $\begin{array}{l}\text { Cooking \& allow to } \\
\text { the vapor }\end{array}$ \\
\hline Verbascum sinaiticum Benth. & Scrophulariaceae & Trnake & $\mathrm{T}$ & Root & Evil sprite & $\mathrm{B}$ & Fumigating \\
\hline Capsicum annuum & Solanaceae & Shirba/berbere & $\mathrm{H}$ & Fruit & Intestinal parasite & $\mathrm{F}$ & Grinding \& eating \\
\hline Lycopersicon esculentum Mill. & Solanaceae & Tsebhi awun & $\mathrm{H}$ & Leaves & Anti leech & $\mathrm{F}$ & $\begin{array}{l}\text { Crushing \& allow to } \\
\text { drink }\end{array}$ \\
\hline Solanum incanum L. & Solanaceae & Engule & $S$ & Root & Amoeba & B & $\begin{array}{l}\text { Crushing \&eating with } \\
\text { enjera honey }\end{array}$ \\
\hline Withania somnifera (L.) Dunal & Solanaceae & Agol & S & $\begin{array}{l}\text { Leave } \\
\text { Stem }\end{array}$ & $\begin{array}{l}\text { Headache } \\
\text { Fibril illness }\end{array}$ & B & $\begin{array}{l}\text { Crushing \& drinking } \\
\text { decoction }\end{array}$ \\
\hline Datura innoxia & Solanaceae & Absho & $\mathrm{H}$ & Stem & Poultry disease* & $\mathrm{F}$ & Pounding with salt \\
\hline Datura stramonium L. & solanaceae & Astenagr/mezrbae & $\mathrm{H}$ & Seed & Teeth ache & $\mathrm{D}$ & $\begin{array}{l}\text { Roasting \& inhalation } \\
\text { of the smoke }\end{array}$ \\
\hline Dombeya torrida & Sterculiaceae & Buwak & $\mathrm{T}$ & Root & Skin wound & $\mathrm{D}$ & pounding \\
\hline Grewia flavescens & Tiliaceae & Mosoqua & $S$ & Bark & Anti-leech & $\mathrm{F}$ & decoction \\
\hline Heteromorpha arborescens & Umbelliferae & Murkus zbi & $S$ & Whole & Common cold & $\mathrm{B}$ & inhalation \\
\hline $\begin{array}{l}\text { Cyphostema adenocaule (Steud. } \\
\text { ex A.Rich.) Desc. ex Wild \& } \\
\text { R.B.Drumm. }\end{array}$ & Vitaceae & Etse zeway & $\mathrm{T}$ & Stem/Root & Snake bite & $\mathrm{F}$ & $\begin{array}{l}\text { Crushing and smearing } \\
\text { the paste }\end{array}$ \\
\hline Balanites aegyptiacus (L.) Delile & Xygophyllaceae & Mekie & $\mathrm{T}$ & $\begin{array}{l}\text { Fruit } \\
\text { Stem } \\
\text { Leaves } \\
\text { Fruits } \\
\text { Branches } \\
\text { Leaves } \\
\text { Leaves } \\
\text { Leaves } \\
\text { Stem } \\
\end{array}$ & $\begin{array}{l}\text {-Abdominal } \\
\text {-Firewood } \\
\text {-Shading } \\
\text {-Food } \\
\text {-Fence } \\
\text {-washing } \\
\text {-detergent } \\
\text {-Fishing } \\
\text {-Forage }\end{array}$ & $\begin{array}{l}F \\
F \\
F \\
F \\
B \\
F \\
F \\
F\end{array}$ & $\begin{array}{l}\text { Crushing, cramming } \\
\text { and squeezing }\end{array}$ \\
\hline
\end{tabular}

Where $\mathrm{T}=$ tree, $\mathrm{S}=$ shrub, $\mathrm{H}=$ herb, $\mathrm{C}=$ Climber, $\mathrm{F}=$ fresh, $\mathrm{D}=$ dry, $\mathrm{B}$, Both $*=$ for animal use only, $* *=$ for both animal human us

\section{Threats to medicinal plants}

Five threats were selected and ten informants were ask to give five for the most threatening factor and one for the least threatening factor in the study area individually based on their thought and observation in their surrounding area. This information was used to determine the highest threats to plants resources in the area. Accordingly Agricultural expansion stood first, and firewood collection free grazing stood second and third respectively as a plant threating factor in Welkait wereda as shown in table2.

Table 2; threats to medicinal plants in the study district

\begin{tabular}{|c|c|c|c|c|c|c|c|c|c|c|c|c|}
\hline \multirow[t]{2}{*}{ Threats } & \multicolumn{10}{|c|}{$\begin{array}{l}\text { Informant (Threat factors put } 1 \text { for least threat and } 5 \text { for the most threat } \\
\text { chronologically based on their severity) }\end{array}$} & \multirow[t]{2}{*}{ Total } & \multirow[t]{2}{*}{ Rank } \\
\hline & I1 & $\mathrm{I} 2$ & I3 & $\mathrm{I} 4$ & I5 & I6 & I7 & I8 & I9 & $\mathrm{I} 10$ & & \\
\hline Free grazing & 4 & 1 & 4 & 4 & 1 & 3 & 4 & 1 & 4 & 4 & 30 & 3 \\
\hline Agricultural & 5 & 5 & 1 & 2 & 5 & 5 & 3 & 2 & 5 & 5 & 38 & 1 \\
\hline Firewood & 2 & 4 & 5 & 5 & 3 & 4 & 2 & 3 & 3 & 2 & 33 & 2 \\
\hline Building & 1 & 2 & 3 & 3 & 4 & 2 & 5 & 4 & 2 & 1 & 27 & 4 \\
\hline Drought & 3 & 3 & 2 & 1 & 2 & 1 & 1 & 5 & 1 & 3 & 22 & 5 \\
\hline
\end{tabular}

Threatened medicinal plants

Ranking of five medicinal plants that report by the informants as threatened in the study area were conduct with 
ten key informants The preferentially selected informants were give the names of five traditional medicinal plant species considered threatened by the community and arrange the plants based on the degree of threat /scarcity by giving 5 for the most threatened and 1 for the least threatened plant species in the study area. More ever, Anogeissus leiocarpa, Terminalia brownie and Securidaca longipedunculata were the three most threatened medicinal plants based on the score given by the informants in table 3 below.

Table 3; Threatened medicinal plants in Welkait wereda

\begin{tabular}{|c|c|c|c|c|c|c|c|c|c|c|c|c|}
\hline \multirow[t]{2}{*}{$\begin{array}{l}\text { Threatened medicinal } \\
\text { plants }\end{array}$} & \multicolumn{10}{|c|}{$\begin{array}{l}\text { Informant (put } 1 \text { for least threatened and } 5 \text { for the most threatened } \\
\text { chronologically based on their severity) }\end{array}$} & \multirow[t]{2}{*}{ Total } & \multirow[t]{2}{*}{ Rank } \\
\hline & $\mathrm{I} 1$ & $\mathrm{I} 2$ & $\mathrm{I} 3$ & $\mathrm{I} 4$ & $\mathrm{I} 5$ & I6 & I7 & I8 & I9 & $\mathrm{I} 10$ & & \\
\hline Acokanthera schimperi & 4 & 2 & 1 & 1 & 2 & 3 & 3 & 1 & 1 & 4 & 22 & 5 \\
\hline $\begin{array}{l}\text { Boswellia papyrifera } \\
\text { (Del.) Hochst. }\end{array}$ & 5 & 1 & 3 & 3 & 3 & 2 & 1 & 3 & 3 & 2 & 26 & 4 \\
\hline $\begin{array}{l}\text { Securidaca } \\
\text { longipedunculata Fresen }\end{array}$ & 1 & 4 & 4 & 4 & 1 & 4 & 4 & 4 & 4 & 1 & 31 & 3 \\
\hline $\begin{array}{l}\text { Terminalia brownii } \\
\text { Fresen. }\end{array}$ & 3 & 3 & 2 & 2 & 4 & 5 & 5 & 2 & 2 & 5 & 33 & 2 \\
\hline $\begin{array}{l}\text { Anogeissus leiocarpa } \\
\text { (DC.) Guill. \& Perr. }\end{array}$ & 2 & 5 & 5 & 5 & 5 & 1 & 2 & 5 & 5 & 3 & 38 & 1 \\
\hline
\end{tabular}

$\mathrm{I}=$ informant

\section{Preference ranking}

Preference ranking were made for five medicinal plants for the most frequently encountered disease (abdominal pain) in the study area and for which they have several alternative plants as readily. Ten favorably selected key informants were shared their knowledge in this exercise. The informants were gave the plants and ask to arrange the five medicinal plants based on their personal preference of usefulness. The medicinal plant that was believed to be the most effective were gave the highest value. Based on that, Solanum incanum is the most preferentially used medicinal plants in threating abdominal pain in the study area as indicated in table 4 . The second and third importantly mentioned medicinal plants in healing the most frequently occurred disease, abdominal pain, are Balanites aegyptiacus and Cucumis dipsaceus respectively.

Table 4; preference ranking of medicinal plants

\begin{tabular}{|c|c|c|c|c|c|c|c|c|c|c|c|c|}
\hline \multirow[t]{2}{*}{ plants name } & \multicolumn{10}{|c|}{$\begin{array}{l}\text { Informant (put } 1 \text { for least important and } 5 \text { for the most important chronologically } \\
\text { based on their curative level) }\end{array}$} & \multirow[t]{2}{*}{$\mathrm{T}$} & \multirow[t]{2}{*}{$\mathrm{R}$} \\
\hline & $\mathrm{I} 1$ & $\mathrm{I} 2$ & $\mathrm{I} 3$ & I4 & I5 & I6 & I7 & I8 & I9 & $\mathrm{I} 10$ & & \\
\hline $\begin{array}{l}\text { Anogeissus } \\
\text { leiocarpa }\end{array}$ & 1 & 1 & 4 & 4 & 1 & 4 & 4 & 4 & 4 & 1 & 28 & 4 \\
\hline $\begin{array}{l}\text { Tamarindus } \\
\text { indica }\end{array}$ & 4 & 2 & 1 & 1 & 2 & 3 & 3 & 1 & 1 & 5 & 23 & 5 \\
\hline $\begin{array}{l}\text { Balanites } \\
\text { aegyptiacus }\end{array}$ & 2 & 3 & 2 & 5 & 5 & 1 & 5 & 4 & 3 & 4 & 34 & 2 \\
\hline $\begin{array}{l}\text { Cucumis } \\
\text { dipsaceus }\end{array}$ & 3 & 5 & 3 & 2 & 4 & 2 & 2 & 3 & 5 & 2 & 31 & 3 \\
\hline $\begin{array}{l}\text { Solanum } \\
\text { incanum }\end{array}$ & 5 & 4 & 5 & 3 & 3 & 5 & 1 & 2 & 4 & 3 & 35 & 1 \\
\hline
\end{tabular}

\section{Matrix ranking}

Based on the relative benefits obtained from each plant, 10 informants were preferentially selected and asked to give value to each attribute on their personal experience and information they have. The list of attributes included medicinal, fire wood, building (construction), charcoal, furniture, edible fruits and fence. The scores were added in order to compare use values of medicinal plants. Matrix ranking of plants used to identify the most multipurpose and the cause for over harvesting of plants. Consequently, Balanites aegyptiacus, Anogeissus leiocarpa and Securidaca longipedunculata were ranked first, second and third most important plant resources in the study district respectively as revealed in the following table 5. 
Table 5; Matrix ranking of medicinal plants

\begin{tabular}{|l|l|l|l|l|l|}
\hline \multirow{2}{*}{ Use value } & \multicolumn{5}{l}{$\begin{array}{l}\text { Informant(put 1for least important use value and 5 for the most important use value } \\
\text { chronologically based on usefulness) }\end{array}$} \\
\cline { 2 - 7 } & $\begin{array}{l}\text { Balanites } \\
\text { aegyptiacus }\end{array}$ & $\begin{array}{l}\text { Anogeissus } \\
\text { leiocarpa }\end{array}$ & $\begin{array}{l}\text { Securidaca } \\
\text { longipedunculata }\end{array}$ & $\begin{array}{l}\text { Acacia } \\
\text { polyacantha }\end{array}$ & Ficus sycomorus \\
\hline Medicinal & 7 & 6 & 7 & 2 & 7 \\
\hline Firewood and charcoal & 5 & 4 & 1 & 4 & 3 \\
\hline Building & 3 & 4 & 3 & 4 & 5 \\
\hline Charcoal & 7 & 7 & 2 & 6 & 3 \\
\hline Furniture & 2 & 1 & 6 & 7 & 4 \\
\hline Edible & 6 & 5 & 4 & 3 & 1 \\
\hline Fence & 4 & 3 & 5 & 1 & 2 \\
\hline Total & 34 & 30 & 28 & 27 & 25 \\
\hline Rank & 1 & 2 & 3 & 4 & 5 \\
\hline
\end{tabular}

Conservation and sustainable use of plant related remedy

According the information obtained from the participated respondents the highest source of traditional medicinal knowledge of plant were from their parents (65\%), and their relatives (20\%), from religious books $(10 \%)$ and $5 \%$ from others sources as indicated in the following figure below. The commitments of the healers to transfer their knowledge to the next generation were assessed. Consequently, $18 \%$ of the respondents were willing to transfer either of their sons or daughters while $52 \%$ of the respondents willing to transfer their knowledge to any of their parent member, $10 \%$ to their relatives and $5 \%$ were not willing to transfer their knowledge to any of their parent member because of anthropological case related with traditional medication system as signposted in figure 4 below. The knockback of the healers to convey their knowledge to the next generation is another controversy for sustainable use and conservation of plant based local community knowledge.

\section{Source of plant related Knowledge}

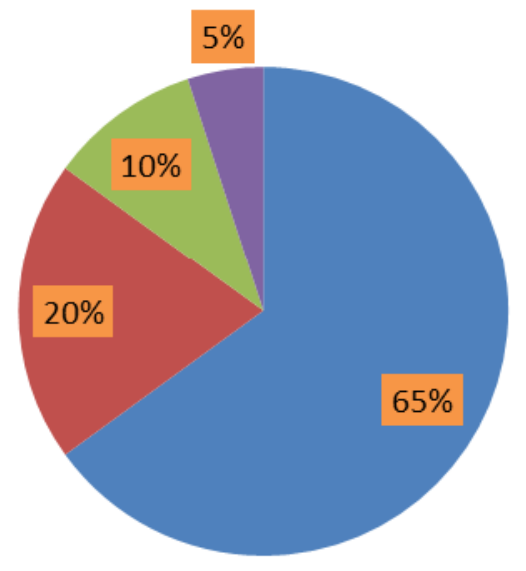

Source of plant related Knowledge from Parent

Source of plant related Knowledge from Relative

- Source of plant related Knowledge from Religious books

Source of plant related Knowledge from Others

Figure 4; source of plant related Knowledge

\section{Correlation of medicinal plant knowledge}

The correlation of age and medicinal plant knowledge was computed to examine the relationships. Consequently, positively significant correlations were observed between age $(\mathrm{p}<0.001)$ and educational level $(\mathrm{p}<0.005)$ of the informants with the number of medicinal plants knowledge by which the informants mentioned. More ever, informants at higher age with no educational level mentioned more medicinal plants than those at lower age and higher educational level as shown in the following correlation table 6 below. The existence of such correlation makes for the continuity of the local community knowledge in remarkable condition. 
Table 6; Correlation of age, gender and educational knowledge of the informants

\begin{tabular}{|c|c|c|c|c|c|}
\hline \multicolumn{6}{|c|}{ Correlations } \\
\hline & & & Age & Gender & Educational level \\
\hline \multirow{3}{*}{$\begin{array}{l}\text { Medicinal } \\
\text { knowledge } \\
\text { informants }\end{array}$} & \multirow{3}{*}{$\begin{array}{l}\text { plant } \\
\text { the }\end{array}$} & Pearson Correlation & $.418^{* *}$ & .122 & $.339^{*}$ \\
\hline & & Sig. (2-tailed) & .004 & .425 & .023 \\
\hline & & $\mathrm{N}$ & 45 & 45 & 45 \\
\hline
\end{tabular}

\section{Acknowledgment}

Wereda and Tabia agricultural experts, the local community administration and respondents are thankful for their incalculable support during the survey. Lastly, but not least Mekelle biodiversity center acknowledged for financing this study.

\section{Reference}

Abebe E., 2011. Ethnobotanical Study on Medicinal Plants used by Local Communities In Debark Wereda, North Gondar Zone, Amhara Regional State. M.Sc. Thesis, Addis Ababa University, Addis Ababa, Ethiopia.

Abera B. Medicinal plants used in traditional medicine by Oromo people, Ghimbi District, Southwest Ethiopia. Ethnobiol Ethnomed. 2014;10:40.

Abiyu Enyew, Zemede Asfaw, Ensermu Kelbessa and Raja Nagappan. 2014. Ethnobotanical Study of Traditional Medicinal Plants in and Around Fiche District, Central Ethiopia. Current Research Journal of Biological Sciences, 6(4): 154-167.

Alebie G, B. Urga, and A. Worku, "Systematic review on traditional medicinal plants used for the treatment of malaria in Ethiopia: trends and perspectives," Malaria Journal, vol. 16, p. 307, 2017.

Alexiades, M. (1996). Collecting ethnobotanical data. In: Alexiades, M.N and Sheldon, J.W. (eds), Selected Guideline for Ethnobotanical Research: A field manual. The New York Botanical garden, Bronex, New York, USA. Pp. 40-102.

Andarge E, Shonga A, Agize M, Tora A. Utilization and conservation of medicinal plants and their associated indigenous knowledge in Dawuro Zone: an ethnobotanical approach. Int J Med Plant Res. 2015;4(3):330-7.

Ashagre M. Ethnobotanical study of medicinal plants in Gui Agro- Pastorilist, Blue Hora district of Borona zone, Oromia region, Ethiopia, M.Sc. Thesis. Addis Ababa: Addis Ababa University; 2011.

Asnake S, Teklehaymanot T, Hymete A, Erko B, Giday M. Survey of medicinal plants used to treat malaria by Sidama people of Boricha District Sidama zone, South Region of Ethiopia. Evid Based Complement Alternat Med. 2016; 10:1155.

Assegid Assefa and Tesfaye Abebe. 2014. Ethnobotanical Study of Wild Medicinal Trees and Shrubs in Benna Tsemay District, Southern Ethiopia. Journal of Science and Development, 2(1): 17-33.

Balick, M.J. and Cox, P.A. (1996).Plants, people and Culture: Science of Ethnobotany. NewYork, USA

Bisht, C. and Badoni, A. (2009). Distribution and indigenous uses of some medicinal plants in Uttarkashi Woreda, Uttarakh and, India. Researcher 1: 38-40.60

Chekole G. Ethnobotanical study of medicinal plants used against human ailments in Gubalafto District, Northern Ethiopia. J Ethnobiol Ethnomed. 2017; 13:55.https://doi.org/10.1186/s13002-017-0182-7.

Chekole G, Asfaw Z, Kelbessa E. Ethnobotanical study of medicinal plants in the environs of Tara-Gedam and Amba remnant forests of Libo Kemkem District, Northwest Ethiopia. J Ethnobiol Ethnomed. 2015;11:4.

Colfer, C. P., Sheil, D. and Kishi, M. (2006). Forests and Human Health: Assessing the Evidence.Center for International Forestry Research, lndonesia.

Cotton, C.M. (1996). Ethnobotany: Principles and Applications. John Wiley and Sons, New York, 412pp.

Centeral Statistical Agency of Ethiopia (CSA, 2007) Census 2007 Tables: Tigray Region, Tables 2.1, 2.4, 2.5 and 3.4

Demel Teketay (2001). Deforestation, Wood famine, and environmental degradation in Ethiopia's highland ecosystems: Urgent Need for Action. NortheastAfrican Council (FSC Africa) 8: 53-76.

Edwars, S., Mesfin Tadesse and I. Hedberg, 1995. Flora of Ethiopia and Eritrea. Vol 2, Part 2, Canellaceae to Euphorbiaceae. Addis Ababa, Ethiopia, Uppsala, Sweden, 456pp

Edwars, S., Mesfin Tadesse, Sebsebe Demissew and I. Hedberg, 2000. Flora of Ethiopia and Eritrea, Volume 2, Part 1, Magnoliaceae to Flacourtiaceae. Addis Ababa, Ethiopia,Uppsala, Sweden, pp: 532.

Getaneh S, Girma Z. An ethnobotanical study of medicinal plants in Debre libanos wereda central Ethiopia. Acad J. 2014:366-79

Gidey M, Beyene T, Yirga G, Signorini AM, Bruschi P. Traditional medicinal plants used by Kunama ethnic group in Northern Ethiopia. J Med Plants Res. 2015; 9(15):494-509.

Hailemariam T, Demissew S, Asfaw Z. An ethnobotanical study of medicinal plants used by local people in the lowlands of Konta Special Woreda, southern nations, nationalities and people regional state, Ethiopia. J 
Ethnobiol Ethnomed. 2009; 5:26.

Hamilton A.C.; Kessy S.P.; Khan, J.A.A.; Lagos-Witte, S. and Shinwari, Z.K. (2003). The Purposes and Teaching of Applied Ethnobotany People and Plants Working Paper 11.WWF, Godalming, UK.

Hanazaki, N.; Souza, V. C. and Rodrigues, R. R. (2006). Ethnobotany of rural people from the boundaries of Carlos Botelho State Park, Sao Paulo State, Brazil. Acta Bot. Bras. 20: 899-909. 62

Hedberg, I. and S. Edwards, (eds.), 1989. Flora of Ethiopia. Volume 3, Pittosporaceae to Araliaceae. Addis Ababa and Asmara, Ethiopia, Uppsala, Sweden, pp: 660.

Hedberg, I., S. Edwards and Sileshi Nemomessa, 2003. Flora of Ethiopia andEritrea. Volume 4, Part I, Apiaceae to Dipsacaceae. Addis Ababa, Ethiopia.

Ibrar, M.; Hussain, F. and Sultan, A. (2007). Ethnobotanical studies on plant resources of ranyal hills, Woreda Shangla, Pakistan. Pak. J. Bot. 39: 329-337.

Kebede A, Ayalew S, Mesfin A, Mulualem G. Ethnobotanical investigation of traditional medicinal plants commercialized in the markets of Dire Dawa city, eastern Ethiopia. J Medicinal Plants Stud. 2016; 4(3):170-8.

Kefalew A, Asfaw Z, Kelbessa E. Ethnobotany of medicinal plants in Ada'a District, East Shewa Zone of Oromia Regional State, Ethiopia. J Ethnobiol Ethnomed. 2015;11:25.

Lal, R. and Junior, W.F. (2011). Where Biodiversity, Traditional Knowledge, Health and Livelihoods Meet: Institutional Pillars for the Productive Inclusion of Local Communities. International Policy Centre for Inclusive Growth, Brazil.

Lamorde M, Tabuti JRS, Obua C, Kukunda-Byobona C, Lanyero H, Byakika- Kibwika P, Bbosa GS, Lubega A, Ogwal-Okeng J, Ryan M, Waako PJ, Merry C Medicinal plants used by traditional medicine practitioners for the treatment of HIV/AIDS and related conditions in Uganda. J Ethnopharmacol. doi:10.1016/j.jep.2010.

Lulekal E, Kelbessa E, Bekele T, Yineger H. An ethnobotanical study of medicinal plants in Mana Angetu District, southeastern Ethiopia. J Ethnobiol Ethnomed. 2008;4(10):1746-429.

Maroyi A. "Traditional use of medicinal plants in southcentral Zimbabwe: review and perspectives," Journal of Ethnobiology and Ethnomedicine, vol. 9, no. 1, p. 31, 2011.

Maryo M, Nemomiss S and Bekele T. 2015. Ethnobotanical study of medicinal plants of the Kembatta ethnic group in Enset based agricultural landscape of Kembatta Tembaro (KT) Zone, Southern Ethiopia. Asian Journal of Plant Science and Research, 5(7):42-61.

Martin, G.J. (1995). Ethnobotany: A method Manual. Chapman and Hall, London. Pp. 265-270

Mesfin F, Demissew S, Teklehaymanot T. An ethnobotanical study of medicinal plants in Wonago Woreda, SNNPR, Ethiopia. J Ethnobiol Ethnomed. 2009; 5:28

Mesfin F, Seta T, Assefa A. An ethnobotanical study of medicinal plants in Amaro Woreda, Ethiopia. Ethnobotany Res Appl. 2014;12:341-54.

Natural Data Base for Africa (NDA) Natural data base, 2008

Rahman A.H.M. "Ethno-botanical survey of traditional medicine practice for the treatment of cough, diabetes, diarrhea, dysentery and fever of santals at Abdullahpur village under Akkelpur Upazilla of Joypurhat district, Bangladesh," Biomedicine and Biotechnology, vol. 1, no. 2, pp. 27-30, 2013

Regassa R. Assessment of indigenous knowledge of medicinal plant practice and mode of service delivery in Hawassa city, southern Ethiopia. J Med Plants Res. 2013;7(9):517-35.

SCBD (2010). Sustainable Forest Management, Biodiversity and Livelihoods: A Good Practice Guide. IUCN, Montreal

Sebukeera, C. (2010). Africa Environment Outlook 2: Forests and Woodlands. Page Address http: //www.unep.org/DEWA/Africa/AEO2 Launch/.Accessed on 12 March, 2013.

Tamene S. An ethnobotanical study of medicinal plants in Wondo Genet natural forest and adjacent kebeles Sidama zone, SNNP region, Ethiopia, M.Sc. Thesis. Addis Ababa: Addis Ababa University; 2011.

Teklehaymanot T, Giday M. Ethnobotanical study of medicinal plants used by people in Zegie Peninsula, northwestern Ethiopia. J Ethnobiol Med. 2007; 3(12):1746-4269.

Thomas, D.W.; Thomas, J. M.; Bromley, W.A.; Mbenkum, F.T. (1989). Korup Ethnobotany Survey: Panda House, Weyside Park, Godalming, Surrey. World Wide Fund for Nature,U.K. 66

Thomas, H. (1995). Indigenous Knowledge, Emancipation and Alination. Journal of knowledge transfer and utilization. 8(1): 63-73. University of Washington.

Woldeab B , R. Regassa, T. Alemu, and M. Megersa, "Medicinal plants used for treatment of diarrhoeal related diseases in Ethiopia," Evidence-Based Complementary andAlternative Medicine, vol. 2018, Article ID 4630371, 20 pages, 2018. 